The proband was seen at 2.5 years of age because of toe walking and severe pes equinovarus for which he had surgery at 2 years. He had bilateral foot drop, atrophy of lower legs, genu recurvatum, and absent deep tendon reflexes. The diagnosis of CMT was also suspected in both parents because of corrected pes cavus and Achilles tenotomies in their teens. Vibration sense was diminished in the toes, motor and sensory NCVs were decreased, and deep tendon reflexes wee absent. The mother's father and a paternal aunt had CMT, and the father's father, his brother, and sister had bilateral pes cavus. On genetic analysis, the boy was heterozygous for both peripheral myelin protein 22 (PMP22) duplication and a mutation in LITAF gene, while each parent had only one mutated CMT gene. (Meggouh F, de Visser M, Arts WFM et al. Early onset neuropathy in a compound form of Charcot-Marie-Tooth disease. Ann Neurol April 2005;57:589-591). (Respond: Dr Frank Bass, Neurogenetics Laboratory AMC, Meibergdreef 9, $1105 \mathrm{AZ}$ Amsterdam, the Netherlands).

COMMENT. A compound phenotype in a severe case of CMT1 is identified by molecular genetic analysis. This more severe phenotype resulted from the co-occurrence of both $P M P 22$ duplication and a LITAF mutation. Modifier genes can alter the severity of CMT caused by PMP22 duplication. In addition to PMP22 and LITAF, 4 other genes (MPZ, $G J B 1, E G R 2$, and $N E F L$ ) have been identified for autosomal dominant demyelinating neuropathies (Young, Suter, 2003; cited by authors).

\title{
GIANT AXONAL NEUROPATHY WITH CNS INVOLVEMENT
}

Linkage and mutation analyses, MRI, EEG and EMG were performed in 6 patients with giant axonal neuropathy (GAN) from 3 consanguineous families examined at Hacettepe University, Ankara, Turkey, and centers in France. All patients had a progressive sensory motor peripheral neuropathy, mental retardation, cerebellar ataxia, pyramidal tract signs, cranial nerve abnormalities, and "frizzly" hair. Onset of symptoms varied from 3.5 to 4.5 years of age. Distal limb weakness was the initial complaint, and 4 patients were wheelchair bound by 9-10 years. Facial diplegia, ptosis, and high forehead were prominent features, 2 female patients had early breast development, and 5 showed scoliosis, pectus carinatum, and pes equino-valgus. Ankle jerks were absent, and pain and light touch sensation impaired. EEGs were abnormal in 3 patients, and EMG abnormalities were consistent with SMAN in 4. MRIs in 4 patients showed diffuse periventricular and cerebellar demyelination and atrophy. Cavum septi pellucidi and vergae abnormalities were also characteristic. Sural nerve biopsy in 1 and skin biopsy in 2 patients showed giant axons and accumulation of whorled filaments in cytoplasm of fibroblasts, respectively. GAN mutations (R293X or a novel mutation, $1502+1 \mathrm{G}>\mathrm{T}$ ) were present in all families, and linkage to chromosome 16q24.1 was confirmed by haplotype analysis. (Demir E, Bomont P, Ersem S et al. Giant axonal neuropathy: clinical and genetic study in six cases. J Neurol Neurosurg Psychiatry June 2005;76:825-832). (Respond: Dr H Topaloglu, Hacettepe Cocuk Hastahanesi, Cocuk Noroloji Bolumu, 06100, Ankara, Turkey).

COMMENT. Giant axonal neuropathy is a recessive neuroectodermal degenerative disorder affecting the peripheral and central nervous systems as well as the skin and hair. These authors and others have located the GAN locus to chromosome 16q24.1, and a homogeneous clinical presentation in 6 patients and 3 families is linked to 2 GAN mutations. 